\title{
Adalimumab-linked reversible lentiginosis
}

\author{
Leonardo Gilardi*, Cecilia Asnal, Patricia Cowan and Inés Morend \\ Centros Ambulatorios OSECAC, Centro Jonas Salk Sarandí, Ciudad Autónoma de Buenos Aires, Argentina
}

\begin{abstract}
Lentigo is a condition marked by the presence of numerous, circumscribed, pigmented maculae, surrounded by normal-appearing skin (lentigines). In psoriatic patients, tumoral necrosis factor alpha (TNF) has been linked to post-inflammatory hyperpigmentation. Treatment with anti-TNF drugs allows the restoration of signaling pathways that favors pigmentation in the psoriatic plaques. We describe a 55 years-old male patient, residing in a subtropical area in the northeast of Argentina, with psoriatic arthritis and no response to conventional drug-modifying drugs. Four weeks after starting anti-TNF treatment with adalimumab, the patient developed lentiginosis in sun-exposed skin areas, which improved with photoprotection and adequate skin humectation, without interrupting specific anti-TNF treatment.
\end{abstract}

As a conclusion, this dermatological adverse event of adalimumab may be easily managed and doesn't preclude anti-TNF treatment.

\section{Introduction}

Lentigo is a condition marked by the presence of numerous, circumscribed, pigmented maculae, surrounded by normal-appearing skin (lentigines). Histological findings include hyperplasia of the epidermis and increased pigmentation of the basal layer. Lentigines have also increased numbers of melanocytes, but these cells do not form nests. [1] Ultraviolet radiation is a major factor that contributes to the generation of acquired lentigines, as indicated by the term "solar lentigo". However, recent epidemiological and mechanistic studies strongly suggest that other environmental factors contribute to lentigines formation. [2]

In psoriatic patients, tumoral necrosis factor alpha (TNF) and interleukin 17 (IL-17) has been linked to post-inflammatory hyperpigmentation. Indeed, recent research has revealed an important role of TNF and IL-17 in the pathogenesis of psoriasis. [3] In contrast, IL-17 enhances the ability of TNF to inhibit melanogenesis, due to low expression of genes critical to pigmentation. As a consequence, treatment with anti-TNF drugs allows the restoration of signaling pathways that favors pigmentation in the psoriatic plaques. [3] So, patients receiving such drugs may have a higher risk of developing such nevi. Lentigines may evolve slowly over years, or they may be eruptive and appear rather suddenly.

\section{Case report}

We describe a 55 years-old male patient, residing in Misiones (a subtropical province in the northeast of Argentina). He has been diagnosed psoriatic arthritis at the age of 15 , without clinical response to conventional drug-modifying drugs. Due to peripheral an axial high activity of the arthritis and extended skin affection (PASI 90), adalimumab treatment was prescribed.

Four weeks after starting anti-TNF the patient improved both dermatological and skeletal symptoms. At the tenth week of treatment hyperpigmented maculae less than $5 \mathrm{~mm}$ diameter were observed in extensive sunlight-exposed areas of head, trunk and limbs (Figure 1). Lesions were disseminated in all skin areas that were previously sunlight exposed and also compromised by psoriasis. He was clinically diagnosed

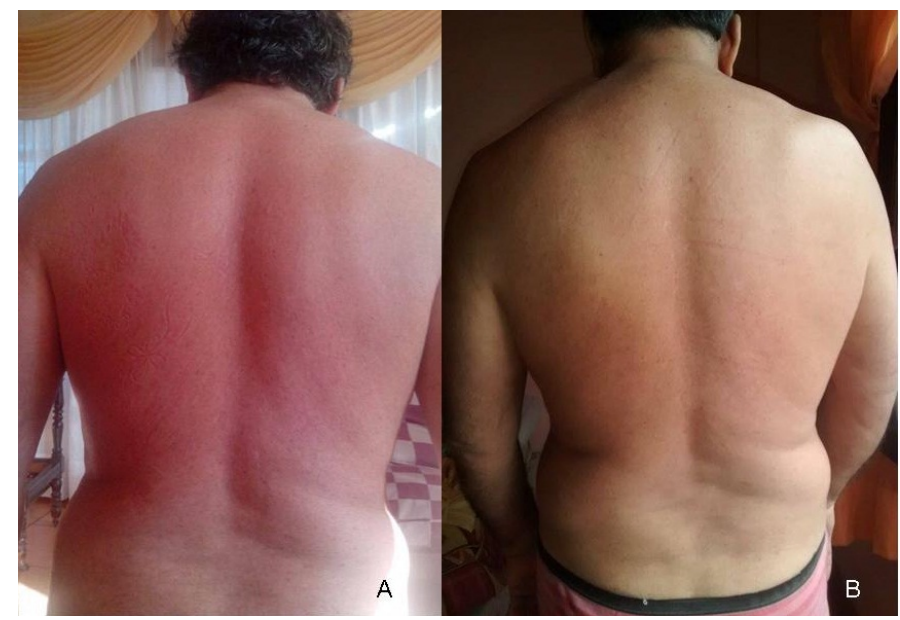

Figure 1. Anti-TNF linked-lentigines. [A] Before and [B] after humectation and photoprotection treatment

as solar lentigo; both photoprotection and repeated humectation of skin were prescribed. Lesions sharply improved and treatment with methotrexate and adalimumab were continued (Figure 1).

\section{Discussion}

Simple lentigo and solar lentigo are benign skin diseases. During natural history of both conditions, hyperpigmented lesions persist even if sunlight exposure is interrupted, so local dermatological treatment is usually required. Lentigines may evolve over years or even decades, or they may be eruptive and appear rather suddenly. Pigmentation may be homogeneous or heterogeneous, ranging from brown to black. Solar

${ }^{\star}$ Correspondence to: Leonardo Gilardi, Sarandí 927, Ciudad Autónoma de Buenos Aires, Argentina, Tel: +541157288859; E-mail: revision_guias@yahoo.com.ar

Key words: adalimumab, lentiginosis, anti-TNF, adverse event

Received: July 20, 2018; Accepted: July 25, 2018; Published: July 30, 2018 
lentigines should be distinguished from ephelides, pigmented actinic keratosis, flat seborrheic keratosis, melanocytic nevus, and malignant melanoma, among other conditions. These can be differentiated based on clinical appearance. [4] The appearance of lentigines must be distinguished from multiple benign eruptive melanocytic nevi, attributed to the immunosuppression produced by biologic drugs. [5]

Lentigo has reported in skin areas that were previously affected by psoriasis in patients receiving different anti-TNF drugs (including infliximab, etanercept or adalimumab). IL-17 and TNF indirectly reduces tyrosinase activity through inhibition of the melanocytestimulating hormone. As a result, excessive levels of both cytokines may induce a greater density of melanocytes, but with suppressed melanogenesis activity. It has been shown that such melanogenic activity recovers quickly after treatment with anti-TNF drugs. [6]

Our patient was residing in a subtropical region, with practically continuous sunlight exposure, but lentigo appeared only after adalimumab treatment. It should be emphasized that lentigines improved with photoprotection and adequate skin humectation, without interrupting specific anti-TNF treatment.

As a conclusion, this rare dermatological adverse event of adalimumab may be easily managed and it doesn't represent a contraindication for anti-TNF treatment.

\section{Authorship}

All authors have made substantive intellectual contributions to this manuscript.

\section{Acknowledgment}

None.

\section{Funding information}

None.

\section{Financial or non-financial conflicts of interests}

None.

\section{References}

1. Hunter J, Savin J, Dahl M (2002) Disorders of Pigmentation. In: Clinical Dermatology. Blackwell Science. 3rd Edition.

2. Nakamura M, Morita A, Seité S, Haarmann-Stemmann T, Grether-Beck S, et al. (2015) Environment-induced lentigines: formation of solar lentigines beyond ultraviole radiation. Exp Dermatol 24:407-411.

3. LaRosa CL, Foulke GT, Feigenbaum DF, Cordoro KM, Zaenglein AL (2015) Lentigines in resolving psoriatic plaques: rarely reported sequelae in pediatric cases. Pediatr Dermatol 32: 114-117.

4. Ortonne JP, Pandya AG, Lui H, Hexsel D (2006) Treatment of solar lentigines. $J$ Am Acad Dermatol 54: S262-271. [Crossref]

5. Costa LA, Belinchón I, Betlloch I, Pérez-Crespo M, Mataix J (2008) Multiple lentigines arising in resolving psoriatic plaques after treatment with etanercept. Dermatol Online J. 14: 1-11.

6. Wang CQ, Akalu YT, Suarez-Farinas M, Gonzalez J, Mitsui H, et al. (2013) IL-17 and TNF synergistically modulate cytokine expression while suppressing melanogenesis: potential relevance to psoriasis. J Invest Dermatol 133: 2741-2752.

Copyright: (C2018 Gilardi L. This is an open-access article distributed under the terms of the Creative Commons Attribution License, which permits unrestricted use, distribution, and reproduction in any medium, provided the original author and source are credited. 